INPLASY

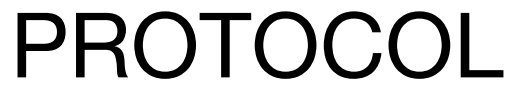

To cite: Jiang et al. Traditional Chinese Medicine for Primary hypothyroidism:A protocol for a systematic review and metaanalysis. Inplasy protocol 202230035. doi:

10.37766/inplasy2022.3.0035

Received: 08 March 2022

Published: 08 March 2022

Corresponding author:

Yong Jiang

jiangyong@pyztcm.com

Author Affiliation:

Chengdu University of

Traditional Chinese Medicine, School of Basic Medical

Sciences.

Support: NSFC Grant No.61801058.

Review Stage at time of this submission: The review has not yet started.

Conflicts of interest:

None declared.

\section{Traditional Chinese Medicine for Primary hypothyroidism: A protocol for a systematic review and meta-analysis}

Jiang, YC1; Zhao, M²; Fan, ZP3; Gan, ZL4; Jiang, Y5.

Review question / Objective: The purpose of this study was to analyze the effect of Traditional Chinese Medicine on Primary hypothyroidism, and to provide evidence for the treatment of Primary hypothyroidism with Traditional Chinese Medicine. Condition being studied: Primary hypothyroidism is a systemic hypometabolic syndrome in which TH deficiency or TH resistance is caused by the disease of the thyroid itself. TH replacement therapy, internationally recognized as the most effective treatment, has many disadvantages. Studies have shown that Traditional Chinese Medicine can effectively eliminate the symptoms of hypothyroidism and improve thyroid secretion. However, the validity of the results was low due to study limitations. Therefore, it is necessary to conduct a systematic analysis of randomized controlled trials of Traditional Chinese Medicine for Primary hypothyroidism.

INPLASY registration number: This protocol was registered with the International Platform of Registered Systematic Review and Meta-Analysis Protocols (INPLASY) on 08 March 2022 and was last updated on 08 March 2022 (registration number INPLASY202230035).

\section{INTRODUCTION}

Review question / Objective: The purpose of this study was to analyze the effect of Traditional Chinese Medicine on Primary hypothyroidism, and to provide evidence for the treatment of Primary hypo- thyroidism with Traditional Chinese Medicine.

Condition being studied: Primary hypothyroidism is a systemic hypometabolic syndrome in which TH deficiency or TH resistance is caused by 
the disease of the thyroid itself. TH replacement therapy, internationally recognized as the most effective treatment, has many disadvantages. Studies have shown that Traditional Chinese Medicine can effectively eliminate the symptoms of hypothyroidism and improve thyroid secretion. However, the validity of the results was low due to study limitations. Therefore, it is necessary to conduct a systematic analysis of randomized controlled trials of Traditional Chinese Medicine for Primary hypothyroidism.

\section{METHODS}

Search strategy: (("Hypothyroidism"[Mesh] OR Hypothyroidisms OR Primary Hypothyroidism OR Hypothyroidism, Primary OR Primary Hypothyroidisms) AND ("Medicine, Chinese Traditional"[Mesh] OR Traditional Chinese Medicine OR Chung I Hsueh OR Hsueh, Chung I OR Traditional Medicine, Chinese OR Zhong Yi Xue OR Chinese Traditional Medicine OR Chinese Medicine, Traditional)) AND ("Randomized Controlled Trials as Topic"[Mesh] OR Clinical Trials, Randomized OR Trials, Randomized Clinical OR Controlled Clinical Trials, Randomized OR RCT).

Participant or population: Participants in the selected articles had no restrictions on age, gender, regional, national, belief, ethnic, sources, and courses of disease.

Intervention: The experimental group was treated with Traditional Chinese Medicine (Traditional Chinese Medicine prescription or proprietary Chinese Medicine) on the basis of conventional western medicine. Prescription drugs require definite dose, but there are no restrictions on drug composition, dosage form and dosage.

Comparator: The control group is treated with conventional western medicine. The specific drugs, dosages, and methods are not limited.

Study designs to be included: We will collect all randomized controlled trials of traditional Chinese medicine in the treatment of primary hypothyroidism. There are no language restrictions.

Eligibility criteria: Types of studies. We will collect all RCTs of TCM in the treatment of $\mathrm{PH}$. There are no language restrictions. Types of participants. Participants in the selected articles had no restrictions on age, gender, regional, national, belief, ethnic, sources, and courses of disease. Types of interventions. There is no requirement for the intervention course, the specific contents of the control group and the experimental group are as follows. Control interventional. The control group is treated with conventional western medicine. The specific drugs, dosages, and methods are not limited. Experimental interventional. The experimental group was treated with TCM (TCM prescription or proprietary Chinese Medicine) on the basis of conventional western medicine. Prescription drugs require definite dose, but there are no restrictions on drug composition, dosage form and dosage.

Information sources: The databases of PubMed, The Cochrane Library, Excerpt Medica Database (Embase), China National Knowledge Infrastructure (CNKI), Wanfang Data Knowledge Service Platform (WANFANG Data), Weipu Information Chinese Periodical Service Platform (VIP), and China Biomedical Literature Service System (SinoMed) will be searched online. The search time is set from the establishment of the search database to March 2022. According to MeSH medical thesaurus, the search terms we selected are "Primary Hypothyroidism", "Hypothyroidisms", "Hypothyroidism, Primary", "Traditional Chinese Medicine", "Traditional Medicine, Chinese", "Clinical Trials, Randomized" and "Trials, Randomized Clinical" etc. Other resources. Additional data can be obtained from references that have been included in the study.

Main outcome(s): Thyroid hormone levels(serum Thyroid stimulating hormone, Total thyroxin, and Free thyroxin, etc.) and symptoms of hypometabolic syndrome 
(including fatigue, weight gain, face, eyelid edema, etc.).

Quality assessment / Risk of bias analysis: As for the literature quality evaluation, we will use the bias risk assessment tool recommended by Cochrane to assess the quality of all included literature and risk of bias. The assessment includes: sequence generation; allocation concealment; blinding of participants, personnel, and outcome assessors; incomplete outcome data; selective outcome reporting; and other sources of bias. The evaluation above would be independently evaluated by 2 researchers. If there are different opinions, we discuss them. If there are still differences exist, we would consult the third appraiser. Otherwise, we need to consult with the Cochrane Professional Group for solution.

Strategy of data synthesis: Relative risk is used as the result index of the binary variable, while standardized mean difference is used as the result index of continuous variable. At the same time, the confidence interval of the combined statistics of continuous variables and the effect indicators of dichotomous variables were set to $95 \%$.

Subgroup analysis: If necessary, the sources of heterogeneity are further analyzed by subgroup analysis.

Sensitivity analysis: If necessary, the sources of heterogeneity are further analyzed by sensitivity analysis.

Country(ies) involved: China.

Keywords: Primary hypothyroidism, Traditional Chinese medicine, Protocol, Systematic review, Meta-analysis.

Contributions of each author:

Author 1 - Yuchang Jiang.

Email: 1985439812@qq.com

Author 2 - Mao Zhao.

Email: 1062844276@qq.com

Author 3 - Zhipeng Fan.

Email: 156183951@qq.com
Author 4 - Zaili Gan. Email: 2356818824@qq.com

Author 5 - Yong Jiang.

Email: jiangyong@pyztcm.com 